\title{
MODELING APPROACH TO SIMULTANEOUS SCHEDULING BATTERIES AND VEHICLES IN MATERIALS HANDLING SYSTEMS
}

\author{
Milorad Vidović ${ }^{1}$, Branislava Ratković ${ }^{2}$ \\ 1,2 University of Belgrade, Faculty of Transport and Traffic Engineering, Vojvode Stepe 305, 11000 \\ Belgrade, Serbia
}

Received 18 November 2014; accepted 17 January 2015

\begin{abstract}
Battery operated handling equipment is the most widely applied concept in materials handling and logistic systems in general. The problem related to its application is in defining the most appropriate scheduling batteries and vehicles to handling tasks. Although the problem can be found in literature very often as very important, solution approaches are very rare and almost don't exist. This paper presents one of possible solving approaches to the problem, considering the optimal assignment of resources (batteries and vehicles) to material handling tasks. Modeling approach proposed is illustrated by a few numerical examples.
\end{abstract}

Keywords: battery powered handling equipment and vehicles, scheduling, assignment, bin packing.

\section{Introduction}

Typical and most widely used solution for the realization of transport and handling processes in any logistics systems (industry, warehouses, sorting and distribution centers) are battery operated handling equipment: forklifts, towing vehicles, automated guided vehicles, etc. There are several reasons for this (Vidovic et al., 1998):

- possibility of free movement of vehicles,

- economy of these vehicles and

- application of electric operated handling equipment represents the best solution from the environmental aspect.

As a confirmation of the wide range of applications of these vehicles, statistics on the number of vehicles delivered of electric operated handling equipment can be used (FEM-IT-ST/N482, May 2013). From this statistics, it can be seen that only in Europe during 2012 number of new electro powered vehicles was around 250000. Wide range of application is articulated by more than 15 years (Wenzl, 1997). The result is, that in the larger industrial and logistics systems 5001000 electro powered vehicles are present, with an average of 1.8-2.1 per vehicle battery, or about 1000-2000 available batteries. Since the batteries serve as the single source of energy for use of these vehicles, a number of questions arise in the domain of appropriate strategy for used batteries replacement, determining the number and characteristics of the batteries in the fleet, as well as concept of battery charging.

Hence, a traditional set of problems inherent in transportation and material handling systems (determining the size and characteristics of the vehicle fleet, the problems of routing, scheduling tasks, etc.) here expands. In this way, the complexity of the traditional optimization problems of material handling systems increases. Vis (2006) presented review the field of design and management of battery-operated automatic guided vehicles (AGV). Author

${ }^{1}$ Corresponding author: mvidovic@sf.bg.ac.rs 
points out that in future studies great concern should incorporate decisions about batteries, and integrate them with routing, scheduling and dispatching decisions.

The importance of the research in this area emphasizes the fact that the price of batteries is very high, often $20-30 \%$ of the price of the vehicle, while the battery life is much shorter than of the vehicle. Battery may be used for approximately 1500 charge cycles, usually 3 or a maximum of 5 years. However, there is a very little research in this area. In the early $80 \mathrm{~s}$, the famous battery manufacturer "VARTA" introduced the concept of "decoupling" vehicles from the battery, accompanied by the so-called "battery management system" and "electro battery powered vehicles management system". It is the system presented at the Hannover Fair 1981 (Preuss, 1987), which involves the formation of the pool of available batteries, which are then "available" for all transport and handling vehicles. Contrary to the conventional approach, the application of this system ensures that batteries are connected with vehicles as a function of the task that vehicles need to realize. This idea, supported by a simple algorithm which is backbone of "electro battery powered vehicles management system”, based on vehicle categories and groups of operating conditions, isn't further developed. Unlike "battery management system" which is, followed by the advancement of electronics, in constant expansion concentrating on the process of optimization battery charging.

In the mid 90s of the last century McHaney (1995) determined the impact of different replacement and battery charging strategies on operating AGVs by using simulation. Author noted that the characteristics of the battery had been usually excluded from the analysis, mainly as a result of incorrect assumptions about minimal impact on productivity and the required number of vehicles as well as insufficient understanding on battery powered vehicles. However, the results of the research and application of simulation models showed that the restrictions introduced by batteries can affect the productivity of vehicles, traffic congestion, increasing the size of the fleet and the cost of the system. Similar conclusions also based on simulation can be found in Ebben (2001), who considered different strategies of replacement and charging batteries. He concludes that it is necessary to keep in mind the restrictions introduced by the batteries, because this can have a serious impact on the performance and cost of logistics system. In short, in the referent literature can be seen a huge gap regarding this field. On one side, there are papers devoted to the problems of operating vehicles in general including battery powered handling transport vehicles. On the other side, there are papers dedicated to electrical or chemical characteristics of the battery and use of these batteries for batterypowered transport-handling vehicles. To the authors best knowledge, the only approach that attempted to observe battery, vehicle, and vehicle task in an integrated manner optimizing their mutual dependencies, is mentioned "VARTA" concept and the paper by Vidovic et al. (1998).

In this paper, the task of optimal vehicles, batteries, and task scheduling is formulated as a modified one-dimensional packing problem with the idea to propose a possible solution which is able to highlight the importance of an integrated approach to solving this class of problems to a greater extent. The paper is organized in following way. In the section two, fundamental

\section{jitte 2}


elements of battery powered vehicles are presented, while the section three gives mathematical formulation of analyzed problem. Numerical example is given in the section four, and finally, concluding remarks are given in the section 5 .

\section{Battery Powered Handling Equipment and Vehicles}

Battery powered handling equipment and vehicles usually use lead-acid battery whose capacity is usually provided per shift, i.e. 5 to 6 hours of moderate exploitation. The reason for the use of lead, rather than nickel-cadmium or lithium-ion battery is primarily economical and reliable production technology. Although lead-acid batteries make $25-50 \%$ of the total mass of the vehicle, while the energy density of all 30$40 \mathrm{Wh} / \mathrm{kg}$. Nickel metal hydride batteries, are considered as outdated technology, while lithium-ion batteries due to its short lifespan and high prices are rarely used. The main advantage of battery-electric vehicles is the lack of harmful emissions and noise reduction because those vehicles are suitable for realization of indoor tasks. Of course, there are also certain disadvantages, particularly those associated with the loss of time required for replacement (5-15 $\mathrm{min}$, and 45 min manually) or charging the battery, which directly influence on the increase of costs and decrease productivity. Charging the lead-acid battery lasts an average of $8 \mathrm{~h}$, while an additional $8 \mathrm{~h}$ is necessary for cooling the battery before using again (Roads $2 \mathrm{HyCom}$, 2007), so that in case of multiple work shifts, extra batteries are needed. The capacity of the battery is between 120-1400 Ah, while the voltages are standardized in the range 24-80V. In practice, electric vehicles usually operate on voltage $40 \mathrm{~V}$ and $80 \mathrm{~V}$. However, although the battery lifespan is around 1500 cycles, battery capacity after 500 to 1000 charge cycles reduced to $60 \%$ or even $50 \%$ from nominal. In addition, the process of discharging the battery during operation affects the reduction of available power for approximately $7.5 \%$, which means that charged and partially emptied battery does not give the same power. For the purpose of facilitating the implementation of tasks on different requirements for energy, available space for battery on the vehicle usually gives opportunity for use of batteries of different capacities. Of course, dimensions and weight of battery must be inside the predefined limits, while the battery voltage must be appropriate.

The working process of the transport and handling vehicles includes a successive implementation of transport and handling operations. One part of the working process is productive and involves moving load, and other non-productive, when empty "movements" are realized. In order to minimize unproductive parts of the process, a large number of optimization algorithms are developed. Transport vehicles with primarily manipulative function (forklifts) are equipped with three electric motors: for moving, lifting and steering, while the vehicles whose main function is transportation (towing vehicles) have own motor for moving and possibly motor for control. Energy consumption during the working process, including the condition of the battery, is a consequence of the configuration of a route, the load weight, vehicle characteristics and the type of task that is being realized (Fig. 1). Determining the energy consumption is one of the most important questions that need to be answered because in that way it is determined the vehicles availability as well the time when the battery must be charged. 


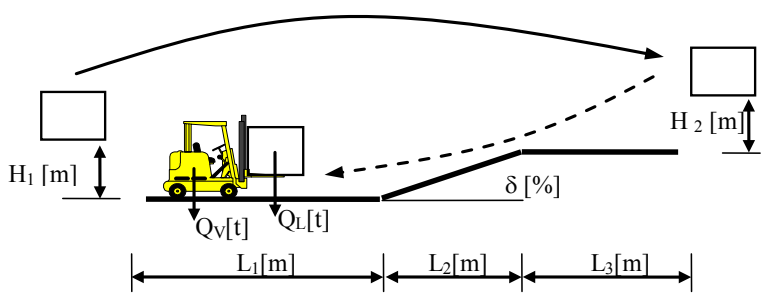

\section{Activity}

Accelerate loaded

Deaccelerate loaded

Reverse movement loaded

Reverse movement empty

Lifting a load

Lowering a load

Lifting empty

Lowering empty
electricity(A)

75

50

35

30

100

80

60

40

Fig. 1.

The Working Process of Transport-Handling Vehicles

There are various different approaches to determining the consumption or battery charge status, although none of them is absolutely accurate: the use of an embedded instrument (ampere-hour meter), the use of statistical data on consumption in realization of various activities and application of analytical calculation methods. Statistic method, by which the battery charge status can be determined precisely, but only after a period of idle and stabilization batteries, is measuring the specific weight of the electrolyte. In this study, we used statistical data about engaged electricity discussed in paper McHaney (1995), partly shown in Fig. 1. Based on this data (duration of individual activities) required energy is easily determined. Another important feature of electro powered vehicles is in way batteries are recharged. There are four possibilities:

- Battery swap, whereby it can be a battery whose application is assigned only to a specific vehicle or battery from the pool which can be used by a group of vehicles with similar characteristics. The first variant is the most frequently used strategy, usually when each vehicle possesses 2-3 spare batteries. This approach is the most economical in terms of battery life, and if the battery is discharged to the extent of $20 \%$ capacity (which represents the depth of discharge
$\mathrm{DOD}=80 \%)$ this represent the optimal working mode.

- Battery charged on vehicle where the vehicle in this period is inactive. It is used only in processes with very low intensity demand.

- Opportunity recharging based on the idea of using a period when the vehicle is idle. In the case when recharging is done before reaching level of $30 \%$ DOD, significantly affect the shortening of battery lifespan. Also, if it is between two fully charging battery charges more than $20 \%$ of its own capacity, lifespan is additionally reduced. It is estimated that this concept shortens the battery lifespan up to $50 \%$.

- Charge rails, similar to the previous strategy, but battery recharges by connecting rails that are under voltage. Rails are installed at suitable locations so that the vehicle can recharged as it moves or, as in the previous case, during the periods of inactivity.

So, it is clear that the exploitation of electro powered vehicles, in addition to problems of vehicle routing, scheduling, dispatching, etc., involves decisions related to use of batteries. This, in turn, raises the need to develop appropriate models, algorithms and decision support systems to help making the most rational vehicles and battery interconnection and coordination. 


\section{Problem Formulation}

In material handling systems in which the handling and transport requests are realized by battery-powered vehicles, the tasks realization assume that vehicles are equipped with batteries whose capacity is large enough to provide realization of assigned tasks. As a result of the different characteristics of load being manipulated, different transport distances and configuration of routes and sites of loading and unloading, tasks impose different requirements in terms of energy and time required for their realization. Hence, optimal realization of the tasks in this case, is the question of optimal allocation of pairs "Battery-vehicle" to transportation and handling tasks.

In other words, if a pair of "Battery-vehicle" is understood as a container - bin, then the problem is in fact the problem of "storing" a certain number of tasks in each of the bins, which corresponds to solving the well known bin packing problem. As in the case of a homogeneous fleet of transport-handling vehicles each unit of transport operation requires equal energy, then the capacity of bins: pairs "Battery-vehicle", correspond to the capacity of the battery attached to the vehicle. In the case of a heterogeneous fleet of vehicles, it is more complicated because the capacity of the pair "Battery-vehicle" must be related to the consumption of energy per unit of transport work of each vehicle. Hence, for the case of battery swap, where batteries are in the pool, and the concept that a one task can be realized with only one vehicle, where vehicle fleet is homogeneous, the simplest version of the scheduling pairs "Battery-vehicle" on tasks (Scheduling and batteries on tasks - SVBT) is possible to formulate as a slightly modified version of the "bin Packing" problem.

$$
\begin{aligned}
& \min _{\text {s.t.: }} \sum_{i \in I} \sum_{j \in J} y_{i j} \cdot c_{j} \\
& \sum_{k \in K} x_{i j k} \cdot e_{k} \leq D O D \cdot y_{j} \cdot E_{j} \quad \forall i \in I \quad \forall j \in J
\end{aligned}
$$

$\sum_{k \in K} x_{i j k} \cdot t_{k} \leq y_{j} \cdot T s h \quad \forall i \in I \quad \forall j \in J$

$\sum_{i \in I} \sum_{j \in J} x_{i j k}=1 \quad \forall k \in K$

$\sum_{j \in J} y_{i j} \leq 1 \quad \forall i \in I$

$$
\begin{aligned}
& \sum_{i \in I} y_{i j} \leq 1 \quad \forall j \in J \\
& y_{i j} \in\{0,1\} \quad x_{i j k} \in\{0,1\} \quad i \in I \quad j \in J
\end{aligned}
$$

where:

I set of available transport and handling vehicles $i \in I$ in fleet

$\mathrm{J}$ set of available batteries $\mathrm{j} \in \mathrm{I}$ in pool

$\mathrm{K}$ set of transport and handling tasks $\mathrm{k} \in \mathrm{K}$ in the system, during observed planning horizon

$c_{j} \quad$ battery costs $j \in J$ (money unit per shift) $\mathrm{E}_{\mathrm{j}} \quad$ available battery capacity $\mathrm{j} \in \mathrm{J}(\mathrm{Wh})$

$e_{k} \quad$ energy engaged during task $k \in K$ realization $(\mathrm{Wh})$

$\mathrm{t}_{\mathrm{k}} \quad$ time need for task $\mathrm{k} \in \mathrm{K}$ realization ( $\mathrm{min}$ ) $\mathrm{T}_{\mathrm{sh}}$ available working hours ( $\mathrm{min}$ )

DOD allowed rate of battery discharge (recommended 80\%) 
$x_{i j k}=\left\{\begin{array}{l}1 \quad \text { if vehicle i uses battery } j \text { when realizing task } k \\ 0 \quad \text { otherwise }\end{array}\right.$ $y_{i j}= \begin{cases}1 & \text { if vehicle } i \text { uses battery } j \\ 0 & \text { otherwise }\end{cases}$

Objective function (1) minimizes the cost of using batteries that are attached to vehicles. Constraint (2) prevents battery discharge below the allowable level of discharge, while constraints (3) limits the use of vehicles across the available working hours. Constraint (4) imposes realization of all tasks in the system, so each task must be realized exactly with one pair "Batteryvehicle". Constraint (5) ensures that on each of the vehicles is attached one battery, while the restriction (6) provides that one battery can be assigned to only one vehicle.

\section{Numerical Example}

Proposed model was tested on five randomly generated numerical examples whose characteristics are shown in Tables 1 and 2 . In the examples, working processes are set to two shifts $\left(T_{s h}=960 \mathrm{~min}\right)$.

\section{Tabel 1}

Task Characteristics

\begin{tabular}{|c|c|c|c|c|c|c|c|c|c|c|}
\hline \multirow{2}{*}{ Task } & \multicolumn{2}{|c|}{ Instance 1 } & \multicolumn{2}{c|}{ Instance 2 } & \multicolumn{2}{c|}{ Instance 3 } & \multicolumn{2}{c|}{ Instance 4 } & \multicolumn{2}{c|}{ Instance 5 } \\
\cline { 2 - 13 } & $\mathbf{t}_{\mathbf{k}}(\mathbf{m i n})$ & $\mathbf{e}_{\mathbf{k}}(\mathbf{W h})$ & $\mathbf{t}_{\mathbf{k}}(\mathbf{m i n})$ & $\mathbf{e}_{\mathbf{k}}(\mathbf{W h})$ & $\mathbf{t}_{\mathbf{k}}(\mathbf{m i n})$ & $\mathbf{e}_{\mathbf{k}}(\mathbf{W h})$ & $\mathbf{t}_{\mathbf{k}}(\mathbf{m i n})$ & $\mathbf{e}_{\mathbf{k}}(\mathbf{W h})$ & $\mathbf{t}_{\mathbf{k}}(\mathbf{m i n})$ & $\mathbf{e}_{\mathbf{k}}(\mathbf{W h})$ \\
\hline $\mathbf{1}$ & 118 & 5664 & 112 & 3472 & 92 & 3588 & 94 & 3384 & 75 & 2175 \\
\hline $\mathbf{2}$ & 104 & 4576 & 95 & 2660 & 110 & 4290 & 30 & 750 & 26 & 884 \\
\hline $\mathbf{3}$ & 16 & 608 & 44 & 1364 & 54 & 2700 & 44 & 1408 & 11 & 462 \\
\hline $\mathbf{4}$ & 94 & 4418 & 32 & 1056 & 81 & 2106 & 107 & 4708 & 54 & 2430 \\
\hline $\mathbf{5}$ & 81 & 3645 & 31 & 961 & 40 & 1040 & 54 & 1350 & 33 & 1023 \\
\hline $\mathbf{6}$ & 63 & 1890 & 103 & 3090 & 115 & 3680 & 54 & 2538 & 19 & 836 \\
\hline $\mathbf{7}$ & 71 & 2414 & 34 & 1666 & 45 & 1530 & 66 & 3300 & 52 & 1352 \\
\hline $\mathbf{8}$ & 14 & 490 & 88 & 3168 & 83 & 2075 & 66 & 2772 & 24 & 864 \\
\hline $\mathbf{9}$ & 81 & 3240 & 114 & 3990 & 90 & 3690 & 61 & 1952 & 13 & 429 \\
\hline $\mathbf{1 0}$ & 85 & 3825 & 102 & 4386 & 113 & 5198 & 36 & 1404 & 87 & 2436 \\
\hline
\end{tabular}

\section{Tabel 2}

Battery Characteristics

\begin{tabular}{|c|c|c|}
\hline Battery & $\mathbf{E}_{\mathbf{j}}(\mathbf{W h})$ & $\mathbf{c}_{\mathbf{j}}$ \\
\hline $\mathbf{1}$ & 7200 & 27 \\
\hline $\mathbf{2}$ & 7920 & 38 \\
\hline $\mathbf{3}$ & 9000 & 22 \\
\hline $\mathbf{4}$ & 10800 & 16 \\
\hline $\mathbf{5}$ & 11160 & 34 \\
\hline $\mathbf{6}$ & 7200 & 31 \\
\hline $\mathbf{7}$ & 7920 & 19 \\
\hline $\mathbf{8}$ & 9000 & 19 \\
\hline $\mathbf{9}$ & 10800 & 31 \\
\hline $\mathbf{1 0}$ & 11160 & 14 \\
\hline
\end{tabular}

Results of application of the proposed mathematical model for the scheduling pairs "Battery-vehicle" on the tasks are shown in Table 3. Implementation of the mathematical model (1) - (7) was implemented in CPLEX 12.2 on Dell Inspiron i5 with 8GB RAM. Times of solving presented instances of the problem are the 6:43, 0:53, 9:27, 8:36 and $0: 39$ seconds, respectively. Of course, these are problems of small dimensions that were designed to test the application of the idea itself, so that the optimum might come in such a short time. It is interesting to note 
that the solution of the problem which does not include battery limitation, but only available working time for vehicles, is to realize all tasks by only one vehicle, and that even such a small case confirms the previously stated observation McHaney (1995) on erroneous assumptions on the minimal impact on battery on productivity and number of battery powered vehicles needed.

Table 3

Results of the Application of Mathematical Models for the Scheduling of Pairs 'Vehicle-Battery' on Tasks

\begin{tabular}{|c|c|c|c|c|c|c|c|c|c|c|}
\hline \multirow{2}{*}{ Task } & \multicolumn{2}{|c|}{ Instance 1} & \multicolumn{2}{|c|}{ Instance 2} & \multicolumn{2}{|c|}{ Instance 3} & \multicolumn{2}{|c|}{ Instance 4} & \multicolumn{2}{|c|}{ Instance 5} \\
\hline & Vehicle & Battery & Vehicle & Battery & Vehicle & Battery & Vehicle & Battery & Vehicle & Battery \\
\hline 1 & 4 & 7 & 10 & 10 & 5 & 8 & 4 & 10 & 5 & 10 \\
\hline 2 & 9 & 8 & 10 & 10 & 5 & 8 & 10 & 4 & 5 & 10 \\
\hline 3 & 10 & 4 & 10 & 10 & 10 & 4 & 9 & 7 & 9 & 4 \\
\hline 4 & 10 & 4 & 10 & 10 & 8 & 10 & 4 & 10 & 5 & 10 \\
\hline 5 & 5 & 10 & 5 & 4 & 8 & 10 & 10 & 4 & 9 & 4 \\
\hline 6 & 4 & 7 & 5 & 4 & 9 & 7 & 5 & 8 & 9 & 4 \\
\hline 7 & 5 & 10 & 5 & 4 & 9 & 7 & 10 & 4 & 9 & 4 \\
\hline 8 & 5 & 10 & 5 & 4 & 10 & 4 & 4 & 10 & 9 & 4 \\
\hline 9 & 9 & 8 & 9 & 7 & 8 & 10 & 5 & 8 & 9 & 4 \\
\hline 10 & 10 & 4 & 9 & 7 & 9 & 7 & 9 & 7 & 9 & 4 \\
\hline Total & \multicolumn{2}{|c|}{4} & \multicolumn{2}{|c|}{3} & \multicolumn{2}{|c|}{4} & \multicolumn{2}{|c|}{4} & \multicolumn{2}{|c|}{2} \\
\hline
\end{tabular}

\section{Conclusion}

The proposed model of optimal scheduling pairs "Battery-vehicle" is an attempt to show the possibilities of integrated approach to optimize electro powered vehicles in the realization of transport and handling tasks. In this paper only one of the segments of the optimization of transport and handling processes that are realized electro powered vehicles. Because it is a complex and very little studied problem, a number of possible extensions and potential directions for future research arise.

In the context of the extension of the proposed model the following options can be made. One extension could be including a heterogeneous fleet of vehicles, of course with appropriate pools of batteries that correspond to certain types of vehicles. Another possible extension of the proposed model could be including a longer period of time in which there is repeated use of the batteries that are during this time recharged, which means including the time for battery replacement. Also, the extension of the proposed model may include the possibility of realizing the same task with larger number of vehicles when tasks need to be treated as sets of partial activities. Thus, for example, the task of unloading the vehicle with ten pallets can be divided into ten activities by unloading a single pallet. In addition to these extensions there are those that would have included additional battery features: losses in power after a certain degree of discharging, the general condition of the battery, which affects the capacity and maximum available power (state of health $\mathrm{SOH}$ ) ... 
etc. In addition very important are the problems of realizing tasks under different battery charging strategies, inclusion of the possibilities of delays in tasks realization, optimal capacity and numbers of batteries determination etc. Finally, optimization of work processes of electro powered vehicles in the realization of transport and handling tasks must involve the development of appropriate heuristic algorithms, to enable solving of real sized practical problems which are much larger.

\section{Acknowledgement}

This work was partially supported by Ministry of Education, Science and Technological Development of the Republic of Serbia through period 2011-2014 (TR36006).

\section{References}

Ebben, M. 2001. Logistic Control In Automated Transportation Networks, Ph.D. thesis, University of Twente, Netherlands. Available from Internet: <http:// doc.utwente.nl/38625/1/t000000a.pdf >.
McHaney, R. 1995. Modelling battery constraints in discrete event automated guided vehicle simulations, International Journal of Production research. DOI: http:// dx.doi.org/10.1080/00207549508904859, 33(11): 3023-3040.

Preuss, P. 1987. Managementsystem für batteriebetriebene Flurförderzeuge. DHF Nr. 1/2. 66-70.

Roads2HyCom. 2007. Project supported by the European Commission's Framework Six program. Available from Internet: <http://www.ika.rwth-aachen. de/r2h/index.php/Main_Page $>$.

Vidović, M.; Sretenović, M.; Cvetić, S. 1998. Genetic algorithm for optimal selection of combination task - vehicle - battery (in Serbian). Soft Computing days. SYM-OP-IS ‘98, Herceg Novi, Montenegro.

Vis, I.F.A. 2006. Survey of research in the design and control of automated guided vehicle systems, European Journal of Operation Research. DOI: http://dx.doi. org/10.1016/j.ejor.2004.09.020, 170(3): 677-709.

Wenzl, H. 1997. Batterien und Flurförderzeuge aus Betreibersicht. Logistikfür Unternehmen: das Fachmagazin der internen und externen Logistik 4-5: 46-51.

\section{jitte 8}

